\title{
TEORÍAS DEL ANÁLISIS Y DISEÑO ORGANIZACIONAL: UNA REVISIÓN A LOS POSTULADOS CONTINGENTES Y DE LA CO-ALINEACIÓN ESTRATÉGICA*
}

\author{
DIEGO ARMANDO MARÍN-IDÁRRAGA** \& JUAN CARLOS CUARTAS-MARÍN**** \\ UNIVERSIDAD JORGE TADEO LOZANO - UNIVERSIDAD AUTÓNOMA DE COLOMBIA
}

Recibido/ Received/ Recebido: 19 /09/2013 - Aceptado/ Accepted/ Aprovado: 19/03/2014

\begin{abstract}
Resumen
El presente artículo realiza una revisión de las teorías de la contingencia estructural y la co-alineación estratégica, las cuales comprenden dos perspectivas medulares en el campo del análisis y diseño organizacional. Se concluye que desde que la teoría de sistemas superó la concepción clásica de la organización insular y cerrada, surgieron diversas corrientes investigativas considerando al ambiente como una de las variables más incidentes en los procesos organizacionales, entre ellas las denominadas teorías del ajuste, que explican a la organización como un fenómeno que se estructura de diferentes maneras según las diversas afectaciones que recibe del entorno y que alcanza su máximo desempeño cuando logra co-alinear sus factores endógenos con los factores situacionales exógenos. Palabras clave: Contingencia estructural, Co-alineación estratégica, Análisis organizacional, Diseño organizacional.
\end{abstract}

\section{ORGANIZATIONAL ANALYSIS AND DESIGN THEORIES: A REVIEW ON CONTINGENT ASSUMPTIONS AND STRATEGIC CO-ALIGMENT}

\begin{abstract}
This article reviews structural contingency and strategic co-alignment theories, which comprise two core perspectives in analysis field and organizational design. It is concluded that the systemic theory exceeded classic conception of insular and closed organization, emerging several current research considering the environment as one of the incident variables in organizational processes, including the so-called adjustment theories, which explain the organization as a phenomenon that it is structured in different ways according to the different effects received from environment and reaches its peak performance when the co-alignment of their endogenous factors with exogenous situational factors is achieved.
\end{abstract}

Keywords: Structural contingency, Strategic co-alignment, Organizational analysis, Organizational design.

Este artículo hace parte del proyecto de investigación titulado "La co-alineación estratégica y su impacto sobre los resultados organizacionales en las Pymes Bogotanas: Análisis comparativo e integrativo en tres sectores económicos", financiado por el Sistema Unificado de Investigación -SUI- de la Fundación Universidad Autónoma de Colombia.

* Doctor (c) en Administración y Dirección de Empresas, Máster en Investigación en Gestión, Universidad Pablo de Olavide de SevillaEspaña; Magíster en Administración y Administrador de Empresas, Universidad Nacional de Colombia. Profesor Asociado II, Programa Administración de Empresas, Universidad Jorge Tadeo Lozano. Director del grupo de investigación AdGeO. Correo electrónico: diego.marin@utadeo.edu.co

*** Máster en Investigación en Gestión, Universidad Pablo de Olavide, Sevilla-España. Especialista en Gestión del Desarrollo Empresarial, Universidad Santo Tomas; Administrador de Empresas, Universidad Nacional de Colombia. Profesor adjunto de tiempo completo, Programa Administración de Empresas, Universidad Autónoma. de Colombia. Integrante del grupo de investigación Observatorio de Relaciones Económicas Internacionales y Administración de Empresas. Correo electrónico: cuartascarlos@hotmail.com 


\title{
TEORIAS DA ANÁLISE E DO DESENHO ORGANIZACIONAL: UMA REVISÃO DOS POSTULADOS CONTINGENTES E DO COALINHAMENTO ESTRATÉGICO
}

\begin{abstract}
Resumo
O presente artigo realiza uma revisão das teorias da contingência estrutural e do coalinhamento estratégico, as quais compreendem duas perspectivas medulares no campo da análise e do desenho organizacional. Conclui-se que desde que a teoria de sistemas superou a concepção clássica da organização insular e fechada, surgiram diversas correntes investigativas considerando ao ambiente como uma das variáveis mais incidentes nos processos organizacionais, entre elas as denominadas teorias do ajuste, que explicam a organização como um fenômeno que se estrutura de diferentes maneiras segundo os diversos impactos que recebe do meio e que atinge seu máximo desempenho quando consegue coalinhar seus fatores endógenos com os fatores situacionais exógenos.

Palavras chave: Contingência estrutural, Coalinhamento estratégico, Análise organizacional, Desenho organizacional.

Marín, D. \& Cuartas, J. (2014) Teorías del análisis y diseño organizacional: una revisión a los postulados contingentes y de la co-alineación estratégica. En: Revista de la Facultad de Ciencias Económicas de la Universidad Militar Nueva Granada. rev.fac.cienc.econ, XXII (1).
\end{abstract}

JEL: M0, M1.

\section{Introducción}

Un ejercicio investigativo en el campo de la Administración, a partir del cual se busquen explicaciones o el entendimiento de su realidad particular, habrá de estar soportado en las teorías de base que incluso puedan derivar en nuevos marcos teoréticos. Como bien lo exponen Christensen \& Raynor (2003), las teorías en el campo de la gestión entrañan la bondad de la comprensión de los fenómenos y su posibilidad predictiva; así que, permiten trascender de la simple categorización en términos de los atributos visibles más obvios del fenómeno -situación de una alta incertidumbre-, hacia correlaciones en términos de las causas que producen ciertos resultados -lo cual significa un avance hacia la predictibilidad-. De modo que la función de la teoría será la del "entendimiento y la predicción” (Fry \& Smith, 1987).

Siguiendo a Sutton \& Staw $(1995,378)$ "la teoría centra su interés en las conexiones entre los fenóme- nos, para explicar por qué se producen los eventos y los pensamientos. Enfatizando en la naturaleza de las relaciones casuales, identifica el origen y orden de los acontecimientos a fin de comprender las razones sistemáticas de su ocurrencia". Así que, las teorías implican presunciones de partida que orientan la búsqueda comprensiva para predecir, explicar y generalizar en un ámbito de imaginación disciplinada (Weick, 1989) ${ }^{1}$. En esta perspectiva, el presente artículo de revisión teórica, asume como importante el acercamiento a las lentes teoréticas para sustentar las hipótesis y orientar los objetivos en las investigaciones empíricas, y se han elegido como unidad de análisis a la teoría de la contingencia estructural y la teoría de la co-alineación estratégica; dos de los referentes más importantes en el campo del análisis organizacional.

La teoría de la contingencia estructural indica que no existe una única manera de configurar la estructura organizacional, pues su diseño depende de la

Weick (1989), plantea que la construcción de teoría se realiza a partir de un proceso de imaginación disciplinada, el cual consiste en un ejercicio de selección artificial con base en prácticas ensayo-error que diseñan, conducen e interpretan experimentos imaginarios. 
influencia de distintos factores contingentes como lo son el ambiente y la estrategia (Donaldson, 2001). Debido a que mucho del trabajo de esta teoría se rezagó en el estudio de interacción entre las variables de estructuración, no dejó muy claro como esas relaciones potencian el desempeño superior. Es por eso que subyace la teoría de la co-alineación estratégica para superar los vacíos dejados por su teoría precedente y explicar que lograr el ajuste o fit entre las variables de estructuración y el ambiente, es una condición imprescindible para alcanzar un mayor desempeño organizacional (Drazin \& Van de Ven, 1985; Venkatraman, 1989).

A pesar de ser dos teorías medulares en el ámbito del análisis organizacional, aun no existen trabajos que las enfrenten resaltando sus características esenciales. Ésta por lo tanto es la brecha que motivó el presente estudio, cuyo objetivo consistió en realizar una revisión teórica de sus principales postulados. Similar al planteamiento metodológico seguido por Zapata \& Mirabal (2013), se realiza una aproximación histórico-hermenéutica con base en una interpretación y análisis diacrónico de las dos teorías, mediante la consulta de revistas científicas y textos especializados. Con este trabajo se realiza un aporte a la teoría de la organización, al entregar un referente que sirva como sustento a estudios futuros sobre todo en los temas del análisis organizacional.

El artículo se divide en tres partes. En la primera se presenta la teórica de la contingencia estructural; en la segunda se expone la teoría de la co-alineación estratégica; en la tercera se introducen las principales conclusiones.

\section{Desarrollo teórico}

Con base en Astley \& Van de Ven (1983), en su clasificación de las escuelas de pensamiento organizacional, tanto a nivel micro (organización individual) como macro (conjunto de organizaciones), se observa un intento taxonómico en las dimensiones del determinismo y el voluntarismo. Para ellos, el determinismo involucra la teorización que se centra en las propiedades estructurales, las cuales están influen- ciadas por las condiciones del ambiente que induce patrones formalizados para orientar el comportamiento en las organizaciones. En cuanto al voluntarismo, lo identifican en aquellas aproximaciones que toman al individuo como la unidad básica de análisis organizacional, en donde se estudian aspectos asociados a sus elecciones individuales, su autonomía y la adhesión e interacción social. De ello, ubican en el determinismo las perspectivas de sistema estructural (nivel micro) y la perspectiva de selección natural (nivel macro). En el voluntarismo clasifican las perspectivas de elección estratégica (nivel micro) y la perspectiva de la acción colectiva (nivel macro).

El presente artículo sigue la clasificación de estos autores en lo atinente al sistema estructural según la orientación determinista en el ámbito micro-organizacional, en donde se ubican la teoría de la contingencia estructural y la co-alineación estratégica (Ilustración 1).

llustración 1. Clasificación de las escuelas organizacionales $^{2}$

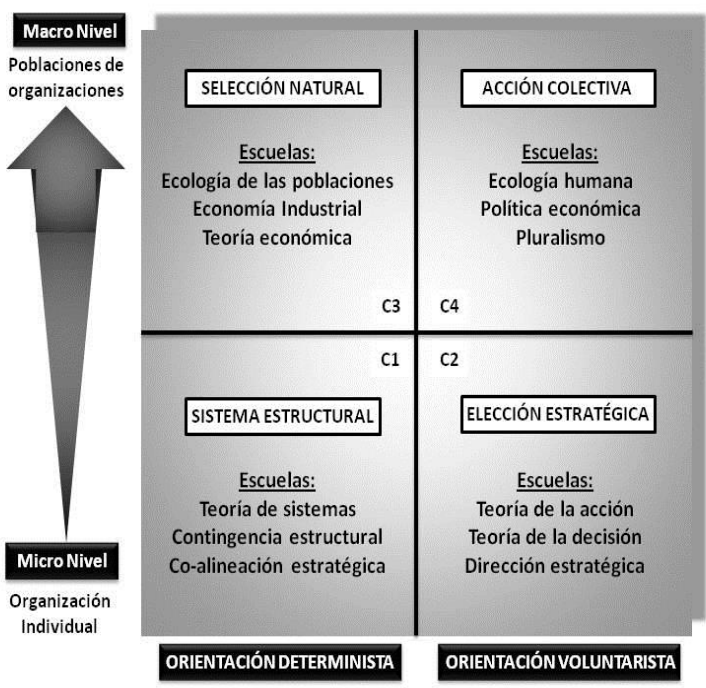

\subsection{La teoría de la contingencia estructural}

La revolución industrial dio lugar al paradigma de la eficiencia en la manufactura emergente del siglo XIX, lo cual suscitó la preocupación por formas or-

2 Fuente: Elaborado con base en Astley \& Van de Ven (1983). 
ganizativas del trabajo que permitieran una mayor producción con un gasto mínimo del esfuerzo humano y de los recursos técnicos y económicos comprometidos. Esta fue la atención inicial de Taylor (1911), quien desarrolló un modelo racional de organización del trabajo y que se constituyó en el origen de la teoría de la organización, la cual centra su análisis, desde una perspectiva normativa, en el fenómeno funcional-mecánico que afecta la eficiencia organizacional (Marín-Idárraga, 2012a). De manera que esta teoría, provee un marco de comprensión de las manifestaciones endógenas de la organización y trata de explicar cómo los procesos, los marcos estructurales y las variables de estructuración influyen en el desempeño (Dalton, Todor, Spendolini, Fielding, \& Porter, 1980; Rapert \& Wren, 1998;), aspecto que Fayol (1949) clarificó, detallando cuáles son esos elementos estructurales y como ellos se integran.

Aunado a esta perspectiva, denominada como la Administración Clásica, el funcionamiento de la sociedad burocrática postulado por Weber (1947) se transcribió a la comprensión de las unidades organizacionales, instaurándose como un modelo ideal para lograr la eficiencia. Posteriormente surgieron diversos aportes que refundaron el planteamiento clásico, con trabajos centrados en clarificar la conformación y funcionamiento de la organización (Sexton, 2005), cuyo denominador común fue la consideración de un sistema cerrado.

Superando la visión determinista clásica de la caja negra, a mediados del siglo XX subyace una apreciación ampliada que asume la existencia de la organización como un sistema abierto, cuya característica esencial es la interacción permanente con el ambiente en donde opera, el cual influye y del cual es influida (Kast \& Rosenzweig, 1972). De este modo surge la teoría de sistemas desde la cual se explican las manifestaciones organizacionales como un conjunto integrado de componentes interactuantes afectados por el entorno del que importan y exportan energía de manera recurrente y recursiva (Katz \& Kahn, 1966).
La concepción de la organización como un sistema que recibe influencias del entorno, permitió que diferentes teóricos se interesaran por analizar tales incidencias sobre el desempeño organizacional, cuyas investigaciones dieron lugar a la teoría de la contingencia. Como lo exponen Kast \& Rosenzweig (1972), la teoría de sistemas sentó las bases para la posterior consideración contingencial de la organización, al establecer la existencia de un supra-ambiente al cual pertenece.

De este modo, queda atrás la perspectiva mecanicista y blindada de la organización postulada por el pensamiento clásico y se instaura el imperativo naturalista, sistémico y situacional de la organización. En esta línea de pensamiento, la teoría contingencial establece que debido a las condiciones ambientales, las organizaciones se diseñan de diferentes maneras en el tiempo de existencia a causa del impacto que reciben desde sus diferentes factores situacionales (Galbraith, 1973). Esto implica que ante diferentes condiciones ambientales, se requerirán diferentes tipos de ajuste estructural (Child, 1972a).

De acuerdo con Donaldson (2001), los factores de contingencia que son influidos por esas condiciones contextuales y que a su vez impactan en el diseño organizacional, son la estrategia, el tamaño, la tecnología y la incertidumbre de tarea. En virtud a que la incidencia recae sobre variables de la estructura organizacional, a este enfoque de estudio se le ha denominada como la teoría de la contingencia estructural.

Los estudios pioneros de esta teoría corresponden a los trabajos acerca de la naturaleza mecánica-orgánica de la organización (Burns \& Stalker, 1961), la influencia de la tecnología (Woodward, 1965; Thompson, 1967; Perrow, 1967), las decisiones de diferenciación-integración (Lawrence \& Lorsh, 1967), las relaciones entre tamaño y diferenciación (Blau, 1970), la formulación de la estrategia (Chandler, 1962) y las investigaciones con respecto a las variables de análisis y diseño estructural planteadas por Hage (1965) y por el denominado grupo Aston ${ }^{3}$

3 El grupo Aston comprendió un equipo de investigación inglés liderado por Derek Pugh, David Hickson y Bob Hinings, quienes realizaron sus investigaciones principalmente en la década de 1960, bajo la óptica de la teoría de la contingencia estructural con conclusiones que ponen énfasis en la condición determinista de la relación ambiente-estructura. 
(Hinnings, Pugh, Hickson, \& Turner, 1967; Pugh et al., 1963; Pugh, Hickson, Hinnings, Turner, 1968).

La premisa fundamental de la teoría de la contingencia estructural es que el ambiente y otros factores situacionales impactan a la organización, por lo que ésta busca ajustar su variables endógenas experimentando siempre diferentes formas de estructuración (Donaldson, 2001). De modo que el postulado taylorista acerca de una mejor manera de administrar (the best way), o el planteamiento de Williamsom (1970) acerca de la forma " $M$ " como la configuración estructural ideal, ceden paso a la máxima contingencial de que "no existe una única forma de organizar; no cualquier forma de organizar es igualmente eficaz" (Galbraith, 1973, 2) .

Burns \& Stalker (1961) iniciadores del enfoque contingente realizaron un estudio con el cual introdujeron una tipología dual de estructuración organizacional. En su enfoque, exponen que según las decisiones de diseño que se asuman en las organizaciones, la estructura tendrá una forma mecánica o una forma orgánica. Por lo tanto plantean que la forma organizacional es el resultado de un proceso racional e intencionado de diseño que da lugar a estructuras rígidas y estáticas propicias para ambientes estables y predecibles o flexibles y adaptables ante ambientes inciertos y hostiles.

La organización tipo mecanicista se caracteriza por una alta estandarización de los procedimientos como resultado de una estricta formalización del comportamiento, sobre todo a través de protocolos y reglamentos por lo general documentados. En estas organizaciones, las decisiones habitualmente están centralizadas en la alta dirección, por lo que la participación de los empleados es baja (McCaskey, 1974; Zapata, Mirabal, \& Hernández, 2009). Se presenta una alta especialización del trabajo debida a una alta diferenciación tanto vertical como horizontal, lo que da lugar a burocracias maquinales con mecanismos de coordinación normalizados según procesos y normas (Mintzberg, 1979).
Por su parte la organización tipo orgánica es aquella en la cual existe una baja formalización ya que los procesos organizacionales deben estar redefiniéndose constantemente; los empleados tienen facultades de participación y la realización de sus tareas es proclive al trabajo en equipo, situación que produce una descentralización de las decisiones y facilita el flujo de conocimiento, motivo por el cual la especialización y la diferenciación vertical tienden a disminuir (Courtright, Fairhurst, \& Rogers, 1989; McCaskey, 1974).

Al encontrar que los diferentes principios de los sistemas de administración clásicos fallaban en explicar el desempeño de las organizaciones en ambientes diferentes, Woodward (1965) buscó justificaciones alternativas. Encontró que los sistemas tecnológicos genéricos bajo los cuales se podía agrupar a las organizaciones, determinaban condiciones de estructuración no identificadas por los clásicos. Concluyó que el diseño organizacional depende del tipo de tecnología adoptada y del grado de incertidumbre en la tarea, de manera que en empresas de producción unitaria (tipo taller), la menor previsión del comportamiento del proceso dará lugar a mayores niveles jerárquicos, en tanto que en empresas de producción mecanizada y automatizada, existirá una mayor previsión del proceso por lo que las jerarquías disminuyen. Así que las organizaciones de manufactura unitaria propiciarán estructuras mecánicas y a medida que se tecnifican surgirán estructuras más orgánicas, y al mismo tiempo, la homologación tecnológica les garantizará un mayor desempeño (Pfeffer, 1982). En la dirección de Joan Woodward, autores como Thompson (1967) y Perrow (1967), con sus diferentes taxonomías, argumentaron que la tecnología se constituye en un imperativo del diseño organizacional. En general, la tecnología influye en la realización de las tareas afectando las destrezas y la discreción del empleado sobre su labor, tornando contingentes la especialización y formalización del trabajo (Pfeffer, 1982).

Para tratar de exponer la complejidad de las organizaciones y la dificultad que entraña elegir formas únicas de estructuración, Lawrence \& Lorsh (1967)

4 De acuerdo con Williamson (1970), la forma "M" corresponde al tipo de estructura Multi-divisional planteada por Alfred Chandler, consistente en la expansión organizacional interna para mejorar la eficiencia, mediante un proceso de integración inverso. 
incluyen una serie de justificaciones que exaltan el carácter contingente del diseño organizativo. Entre ellas establecen que: (i) la organización es un sistema abierto conformado por una serie de partes que se subdividen y que luego en su operatividad deben acoplarse para buscar su adaptación al ambiente externo; (ii) la subdivisión del sistema y la necesidad de ajuste llevan al estudio de las variables de diferenciación (subdivisión jerárquica y funcional) e integración (coordinación); (iii) la existencia de un mayor grado de diferenciación (sobre todo en las empresas más grandes), propiciará conflictos periódicos, lo que supondrá un mayor esfuerzo de integración, a partir de modos alternativos que superen las visiones tradicionales de los clásicos (quienes suponían que tal integración se lograba a través de un proceso racional y mecánico mediante la influencia de la jerarquía directiva); (iv) las múltiples y diversas condiciones externas a la organización, van a requerir características organizativas disimiles; y (v) el diseño de una organización que responde a las demandas ambientales, habrá de considerar además la posibilidad de satisfacer las necesidades de realización y competencia de los individuos.

Dentro de los factores contingentes que influyen en el diseño organizacional, el tamaño ha sido considerado como una de las principales variables a analizar y controlar en los estudios de diseño organizacional. Blau (1970), introdujo la variable tamaño como incidente de la forma estructural, y concluyó que a medida que las empresas crecen hay mayor presión hacia la diferenciación jerárquica y funcional, lo que a su vez conlleva mayores niveles de formalización a causa de mayores exigencias por la descentralización. Este aspecto fue corroborado en las investigaciones de Meyer (1971) quien concluyó que el tamaño era el ocasionante de una mayor diferenciación organizacional y no al contrario, y del mismo Meyer (1972) quien argumentó que el tamaño promovía la instauración y variación de mecanismos de control organizacional, así que a mayor tamaño hay una relación positiva con la especialización y la formalización y negativa con la centralización (Child, 1972b; 1973). Por lo tanto, según Mintzberg (1979), cuanto más grande sea la organización, más compleja será su estructura, en términos de más especialización de tarea, más unidades funcionales diferenciadas y más desarrollado su componente administrativo ${ }^{5}$.

Otro de los factores contingentes de gran relevancia en los estudios organizacionales es la estrategia. Su análisis ocupó igualmente la atención de la teoría contingencial, al considerar que los impactos ambientales se ven primeramente reflejados en la estrategia de la organización, cuya reformulación conlleva inexorablemente un rediseño estructural. El primero de esta corriente en advertirlo fue Chandler (1962), quien desde una perspectiva histórica, investigó cuatro grandes empresas norteamericanas (Sears Roebuck, Standard Oil, Du Pont y General Motors) desde finales del siglo XIX y hasta mediados del siglo XX, observando que a medida que las empresas crecían y diversificaban su portafolio de productos, proporcionalmente tendían a desarrollar estructuras multidivisionales que trascendían las formas funcionales clásicas ${ }^{6}$. Sus trabajos popularizaron la conocida tesis "la estructura sigue a la estrategia", al mostrar que en contextos donde se eligen estrategias diversificadas, la forma tradicional de poder centralizado termina siendo ineficiente, por lo que el paso de la forma " $U$ " (unitaria) a la forma " $M$ " (multidivisional) es inevitable (Williamson, 1970).

Por lo tanto, se concluye la existencia de una relación intrínseca y correspondiente entre la estrategia y la estructura. Esta tesis fue corroborada por la misma época en los trabajos de Rumelt (1974), quien observó que a partir de 1940 se produjo un aumento importante en el número de empresas diversificadas y con formas estructurales por divisiones y con las investigaciones de Channon (1973) y Dyas \& Thang-

5 La medición del componente administrativo fue introducida como un indicador de intensidad administrativa "IA" que correlaciona la cantidad de personal administrativo "A" con el personal no administrativo ocupado "P" mediante IA= AA+P (Anderson \& Warkov, 1961; Tosi \& Platt, 1967).

6 De acuerdo con Mintzberg (1979), la forma multidivisional comprende una configuración estructural con unidades estratégicas de negocio semi-autonomas acopladas mediante un componente administrativo central. 
heiser (1976) quienes demostraron que a lo largo del tiempo la relación estrategia-estructura se afianza pues las empresas que optan por la diversificación producen una transformación hacia la estructura multi-divisional.

Otros estudios en la teoría de la contingencia estructural centraron su atención en las variables endógenas del diseño y su implicación para el desempeño organizacional. El origen de esta línea de trabajo con foco en el análisis de la estructura organizacional se remonta a las investigaciones del denominado grupo Aston en Inglaterra, cuyos resultados fueron publicados de manera sucesiva en la década de 1960 principalmente en la revista Administrative Science Quarterly (Hinnings et al., 1967; Pugh \& Pheysey, 1972; Pugh et al., 1968; Pugh, Hickson, Hinnings, 1969a, 1969b; Pugh et al., 1963).

Siguiendo la línea de la burocracia weberiana y convencidos de la legitimidad de la investigación empírica, su preocupación fue el análisis de las variables internas que deben atenderse para crear una estructura coherente con los diferentes factores de contingencia (Hall, 1962). Integrando interdisciplinariamente desarrollos de la sicología, la sociología, la economía y la política con la teoría organizacional, los aportes del grupo Aston se enmarcan dentro del denominado determinismo contextual, y la conclusión central es que existen una serie de regularidades entre las variables de estructuración y el contexto de tarea cuya articulación afecta el desempeño organizacional. Así, las interacciones entre la concentración de la autoridad y la necesidad de control sobre el flujo operacional, darán lugar a la estructuración de las actividades (Pugh et al., 1968). Para alcanzar el control del trabajo, emergen una serie de mecanismos que Pugh et al. (1963) identifican como las seis dimensiones primarias de la estructura organizacional: i) especialización, correspondiente a la división del trabajo dentro de la organización que da lugar a la diferenciación funcional, ii) estandarización, referida a la normalización de los procesos operativos y de gestión iii) formalización, referente a la definición de rutinas y normas documentadas iv) centralización, concerniente al locus de la autoridad para tomar de- cisiones, v) configuración, relativa a la definición de la posiciones jerárquicas que median las relaciones entre directivos y subordinados (conocida como tramo de control); y (vi) flexibilidad, entendida como la magnitud y frecuencia del cambio organizativo.

A pesar de las críticas de las que ha sido objeto el trabajo del grupo Aston (Child, 1972b; Grinyer \& Yasai-Ardekani, 1980; Reiman, 1973), en especial por la falta de claridad en como la articulación de las variables estructurales producen un desempeño superior (Fry, 1982; Schoonhoven, 1981), vale reconocer que sus hallazgos han sido fundantes de la teoría de estructuración de las organizaciones y han orientado trabajos posteriores sobre todo en el campo del diseño organizacional (Mintzberg, 1979; Galbraith, 1973).

Otra perspectiva genealógica de la teoría de la contingencia estructural, paralela y concordante con el pensamiento del grupo Aston, fue la postulada por Hage (1965), quien propuso un enfoque axiomático para la estructura organizacional, con base en una serie de proposiciones a partir de ocho variables clasificadas según los significados y fines de la organización. Este autor expuso que el significado organizacional se refiere a la división del trabajo y su coordinación y bajo su lente conjuntó cuatro variables: (i) la complejidad, referente a la especialización de tareas; (ii) la centralización, atinente a la proporción de trabajadores que participan en la toma de decisiones; (iii) la formalización, correspondiente con la discrecionalidad permitida al trabajador según la codificación del trabajo; y (iv) la estratificación, relativa a las posiciones jerárquicas o diferenciación vertical. Por su lado, los fines organizacionales representan los estados previstos de alcance y agrupan a (i) la adaptabilidad -cambio estructural-; (ii) la productividad -rendimiento operacional-; (iii) la eficiencia racionalización de costos-; y (iv) la satisfacción en el trabajo -moral de los trabajadores-.

Hage (1965) siguiendo el postulado de Burns \& Stalker (1961), asoció su axiomática conforme a las organizaciones tipo mecánico y orgánico según se específica en la Tabla 1. 
Tabla 1. Dos tipos ideales de organizaciones prescritas por la teoría axiomática ${ }^{7}$

\begin{tabular}{|c|c|}
\hline $\begin{array}{c}\text { Modelo orgánico } \\
\text { (Énfasis en la adaptabilidad) }\end{array}$ & $\begin{array}{c}\text { Modelo mecanicista } \\
\text { (Énfasis en la producción) }\end{array}$ \\
\hline Alta complejidad & Baja complejidad \\
Baja centralización & Alta centralización \\
Baja formalización & Alta formalización \\
Baja estratificación & Alta estratificación \\
Alta adaptabilidad & Baja adaptabilidad \\
Baja producción & Alta producción \\
Baja eficiencia & Alta eficiencia \\
Alta satisfacción en el trabajo & Baja satisfacción en el trabajo \\
\hline
\end{tabular}

Todo lo descrito anteriormente resume el trabajo germinal de los diferentes autores inscritos en la corriente de la contingencia estructural, que iniciaron una perspectiva de pensamiento dominante en el campo de la teoría organizacional. Las investigaciones de partida sentaron las bases para la comprensión y explicación del fenómeno de estructuración organizacional en las investigaciones siguientes y hasta la fecha, a la luz del postulado que no existe una única manera de estructurar la organización, pues las formas organizacionales están sometidas a las influencias situacionales de los factores contingentes. Pese a las críticas que la teoría de la contingencia estructural ha recibido y las cuales Pfeffer (1982) resume como una cierta ambigüedad conceptual y de medición, su constructo aún es un punto de referencia de los estudios en este campo y sigue soportando estudios recientes (Fegh-hi, 2010; Liao, Chuang, \& To, 2011; Menguc \& Auh, 2010; Sahay \& Gupta, 2011; Santra $\&$ Giri, 2008).

\subsection{La teoría de la co-alineación estratégica}

La teoría tradicional-ortodoxa de la organización planteaba asociaciones causales de las variables que afectan a la organización en el modo que una influencia $X$ produce siempre un efecto $Y$ (e.g. las teorías funcionales y las teorías conductuales de las necesidades). Con los trabajos de la teoría de la contingencia, las variables se influyen de modo situacio- nal, así que un ajuste entre dos variables afectará a terceras variables (Dewar \& Werbel, 1979; Schoonhoven, 1981; Venkatraman, 1989). Precisamente la idea contingente postula la imposibilidad de encontrar una única forma de estructurar la organización, dado que el desempeño estará influenciado por las diferentes circunstancias de ajuste entre las variables endógenas y los factores externos (Donaldson, 2001). De acuerdo con Sillince (2005), sobre esta base emerge la teoría de la co-alineación estratégica, -denominada también teoría del fit o teoría de la congruencia organizacional- al considerar que (i) existe una asociación entre factores contingentes y la estructura de la organización, (ii) esos factores contingentes inciden en la estructura y (iii) la estructura busca ajustarse a los factores contingentes para alcanzar un alto desempeño, sobre todo a partir de la congruencia entre ambiente, estrategia y estructura.

En este marco, la teoría de la congruencia organizacional (o co-alineación estratégica) trasciende a la teoría de la contingencia estructural, para indicar que el desempeño no aparece por la mera inclusión en el diseño organizativo de variables estructurales inducidas por el ambiente, sino que además esas variables deben ser objeto de un ajuste estratégico que potencie un mayor desempeño (Ensign, 2001).

Los orígenes de esta teoría se remontan a los trabajos iniciales de Drazin \& Van de Ven (1985), Donaldson (1987) y Fry \& Smith (1987) en el ámbito organizacional y a Venkatraman \& Camillus (1984) y Venkatraman (1989) en el campo de la dirección estratégica. Los rasgos comunes de las diferentes aproximaciones son la consideración de un estado de congruencia necesario denominado ajuste o fit en su acepción inglesa, y que ha sido asumido como un simbolismo lingüístico para explicar la sincronía, consistencia o coherencia requerida entre los factores situacionales, los parámetros estructurales endógenos y el desempeño organizacional, en una perspectiva holística e integrada -autores como Nadler \& Tushman (1999) han optado por llamarlo congruencia-.

A partir de las críticas que recibió la teoría de la contingencia estructural, sobre todo por su falta de cla-

7 Fuente: Hage (1965). 
ridad acerca de cómo la estructura organizacional se ajusta con los factores contingentes para garantizar un mayor desempeño (Fry, 1982; Schoonhoven, 1981), diversos académicos se interesaron en cubrir esos vacíos, con la postulación de modelos teóricos que sustentaron los estudios posteriores (Donaldson, 1987; Drazin \& Van de Ven, 1985; Venkatraman \& Camillus, 1984; Venkatraman, 1989). Esos autores coinciden en exponer que las organizaciones enfrentan dos decisiones básicas: (i) definir las condiciones organizacionales que permitan a la empresa su mejor alineación con los factores de contingencia externos (ajuste exógeno) y (ii) articular esas variables internas mediante estrategias y procesos que permitan su congruencia (ajuste endógeno). La tesis medular es que el desempeño superior subyace cuando la organización es capaz de integrar ambas condiciones de ajuste; la interna y externa.

En esa dirección, Venkatraman \& Camillus (1984), proponen un esquema teórico para comprender el ajuste estratégico consistente en dos condiciones; de un lado la conceptualización del ajuste y de otro lado el dominio del ajuste. Estas dos condiciones dan lugar a una matriz con seis celdas atendiendo a las dimensiones del contenido del ajuste y del proceso del ajuste, en consonancia con las diferentes corrientes de investigación estratégica. En la primera dimensión definen tres modalidades de ajuste: (i) fit externo/contenido, correspondiente a la alineación de la estrategia con las condiciones ambienta- les -escuela de la formulación de la estrategia; (ii) fit interno/contenido, referido a la adaptación de los mecanismos administrativos y organizacionales en línea con la estrategia -escuela de la implementación de la estrategia-; y (iii) fit integrado/contenido atinente a la gestión estratégica que implica tanto la formulación como la puesta en marcha de la estrategia y que cubre a las decisiones organizacionales y ambientales. En la segunda dimensión incluyen las restantes tres modalidades de ajuste: (i) fit externo/ proceso, relativo al balance de las interrelaciones entre las estrategias de varias empresas que colectivamente compiten por la asignación de recursos -escuela de las redes inter-organizacionales-; (ii) fit interno/proceso, relacionado con la discreción gerencial para equilibrar las distintas decisiones relacionadas con los mecanismos organizacionales escuela de la elección estratégica-; y (iii) fit integrado/proceso, correspondiente a la configuración no causal de la organización con su medio ambiente - escuela de la gestalt ${ }^{8}$ - (Tabla 2).

Por su parte, Drazin \& Van de Ven (1985) exponen que es posible asumir formas de acople de la organización en atención a las configuraciones estructurales dependientes del ambiente en que opera la empresa. De este modo plantean como perspectivas de análisis: (i) la selección, que asume una congruencia entre las variables estructurales endógenas y las condiciones ambientales, pero sin implicaciones para el desempeño; (ii) la interacción, que establece qué

Tabla 2. Marco conceptual para comprender las diferentes perspectivas de ajuste ${ }^{9}$

\begin{tabular}{|c|c|c|c|}
\hline \multirow{2}{*}{$\begin{array}{c}\text { Contenido del ajuste } \\
\text { (Criterios que se interrelacionan } \\
\text { con la estrategia) }\end{array}$} & \begin{tabular}{c} 
Conceptualización del Ajuste \\
\cline { 2 - 3 }
\end{tabular} & $\begin{array}{c}\text { Proceso del ajuste } \\
\text { (La estrategia como criterio de interrelaciones) }\end{array}$ \\
\hline \multirow{2}{*}{ Dominio del ajuste } & Externo & Formulación de la estrategia & Redes inter-organizativas \\
& Interno & Implementación de la estrategia & Elección estratégica \\
& Integrado & Formulación e implementación de la estrategia & Gestalt \\
\hline
\end{tabular}

8 De acuerdo con Veliyath \& Srinivasan (1995), la gestalt comprende una entidad organizada o el "todo" en el cual las "partes", aunque diferenciadas entre sí, son interdependientes y adquieren características propias producto de su inclusión en el "todo", el cual a su vez posee otras características particulares que no comparte con ninguna de las "partes". Por ejemplo la organización como un "todo" posee diferentes arreglos estructurales y diversos patrones de desempeño, lo cual es una característica de la co-alineación.

9 Fuente: Adaptado de Venkatraman \& Camillus (1984). 
influencias reciprocas y bivariadas entre el ambiente y la estructura, entrañan un potencial generador de impactos en el desempeño organizacional; y (iii) la sistémica, que reconoce que el ajuste se da a partir del análisis de diversas variables estructurales y del entorno, para producir diversas formas de desempeño y múltiples diseños organizativos igualmente efectivos (Tabla 3).

Donaldson (1987) introdujo otro modelo comprensivo de co-alineación estratégica que denominó SARFIT (Structural Adapatation to Regain Fit), el cual establece la existencia de un ajuste entre cada factor de contingencia y uno o más aspectos de la estructura organizacional, tal que el fit afectará positivamente el desempeño en tanto que el misfit afectará negativamente el desempeño. Un cambio en un factor de contingencia (e.g. tamaño o grado de diversificación de producto), conduce a un desajuste entre la estructura existente y el nuevo valor que asume el factor contingente (e.g. el nuevo tamaño afecta la especialización del trabajo o la nueva diversificación de producto da lugar a una configuración multi-divisional).

El desajuste producido conlleva comportamientos disfuncionales en la organización -como una inadecuada toma de decisiones, pérdida de la comunicación y desmotivación entre otros-, que ocasionan un bajo desempeño manifestado por ejemplo en pérdida de oportunidades para alcanzar mayores ventas $y$ en baja rentabilidad para los accionistas. Este bajo desempeño suele presentarse más rápidamente en contextos de negocio hostiles, en donde las empresas no cuentan con recursos disponibles para apalancar el rendimiento deseado sin necesidad de cambiar la estructura. Ahora, es probable que el bajo desempeño conduzca a un cambio estructural ante un ambiente iliberal que ejerce un efecto moderador ${ }^{11}$. La nueva estructura organizacional produce un ajuste sostenido hasta un nuevo cambio en los factores de contingencia haciendo que el ciclo se repita (Ilustración 2).

Ilustración 2. Modelo SARFIT12

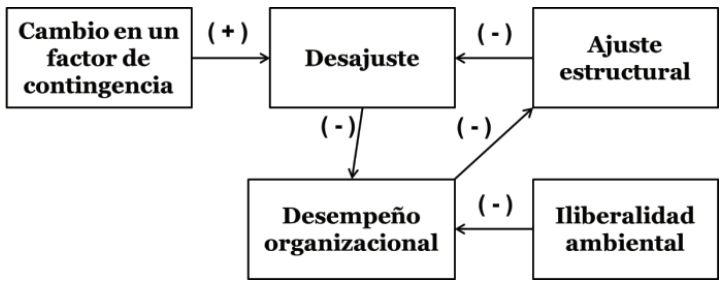

En otra idea, Venkatraman (1990) define la co-alineación como una metáfora para representar el ajuste entre diferentes dimensiones teóricas. Sin embargo el autor considera que esta concepción deriva en una dificultad a la hora de realizar su medición dada la complejidad de los constructos que se buscan testear empíricamente. En este sentido, propone tres alternativas metodológicas: (i) la perspectiva interaccionista, con base en análisis de moderación entre las variables, (ii) la perspectiva de desviación de un

Tabla 3. Ajuste en términos de selección, interacción y sistemas ${ }^{10}$

\begin{tabular}{|c|c|c|c|}
\hline $\begin{array}{c}\text { Concepto } \\
\text { del ajuste }\end{array}$ & Selección & Interacción & Sistemas \\
\hline Visión Inicial & $\begin{array}{c}\text { El ajuste es asumido como una } \\
\text { premisa subyacente entre la } \\
\text { estructura y el ambiente }\end{array}$ & $\begin{array}{c}\text { El ajuste es asumido como la incidencia } \\
\text { bivariada entre pares de factores ambiente- } \\
\text { estructura que afectan el desempeño. }\end{array}$ & $\begin{array}{c}\text { El ajuste es la consistencia interna de múltiples } \\
\text { factores de contingencia y diversas variables } \\
\text { estructurales que afectan el desempeño de } \\
\text { diferentes maneras. }\end{array}$ \\
Visión futura & $\begin{array}{c}\text { El ajuste es concebido como la } \\
\text { selección natural (o por influencia } \\
\text { gerencial), en un nivel macro- } \\
\text { organizacional }\end{array}$ & $\begin{array}{c}\text { El ajuste corresponde al análisis residual } \\
\text { de la relación lineal entre el ambiente y la } \\
\text { estructura }\end{array}$ & $\begin{array}{c}\text { El ajuste es la consistencia interna logrado } \\
\text { como resultado de la equifinalidad (múltiples } \\
\text { configuraciones estructurales con el ambiente } \\
\text { para lograr el objetivo de desempeño). }\end{array}$ \\
\hline
\end{tabular}

10 Fuente: Drazin \& Van de Ven (1985).

11 Donaldson (1987) retoma el concepto de ambiente iliberal postulado por Child (1972a) para describir a un contexto de alta intensidad competitiva.

12 Fuente: Donaldson (1987). 
perfil, que evalúa la congruencia entre las variables según su adherencia a un perfil especificado a priori y externamente por el investigador, y c) la perspectiva de co-variación, que evalúa la consistencia interna de las variables a través de un modelo estructural.

Las anteriores perspectivas son consideradas como los aportes iniciales de la teoría de la contingencia estructural y con referencia a ellos, se ha desarrollado una diversidad importante de estudios. Dentro de la amplia producción investigativa, contemporáneamente vale resaltar dos modelos: (i) el modelo de la congruencia del comportamiento organizacional de Nadler \& Tushmann (1997) y (ii) el modelo de la multi-contingencia organizacional de los autores Burton \& Obel (2004).

Nadler \& Tushmann (1997) plantean un esquema como fruto de su trabajo investigativo por más de una década, el cual refleja los conceptos esenciales de los sistemas abiertos (Kast \& Rosenzweig, 1972) pero con una mayor especificidad. En él se detallan los insumos críticos -inputs-, los procesos de transformación -throughput-y los productos principales -outputs- que caracterizan el funcionamiento de la organización. El modelo enfatiza en los procesos de transformación mediante una definición de las variables críticas del sistema de interdependencias, ati- nentes tanto a la organización formal como informal, y establece que la congruencia entre ellas garantizará un mejor desempeño. Esas variables críticas son cuatro: estructura y procesos informales, disposiciones de la organización formal, personas y trabajo. Para los autores la congruencia corresponde al grado en el cual las necesidades, exigencias, objetivos y estructuras son compatibles entre las cuatro variables críticas (e.g. tareas a desarrollar e individuo responsable de su realización), para lo cual los gerentes deben enfrentar tres retos básicos: (i) la adaptación estratégica; tomar las decisiones apropiadas referidas a la interacción con el ambiente, (ii) la adaptación estrategia-organización; moderar la arquitectura a la estrategia, y (iii) la adaptación endógena; procurar el equilibrio entre las cuatro variables críticas (Ilustración 3).

Burton \& Obel (2004) desarrollan una teoría comprensiva del diagnóstico y diseño organizacional con sustento en el fit estructural. Esta teoría representa una síntesis integradora de las teorías funcional y contingencial y se fundamenta en la premisa que la organización es una entidad que procesa información, cuyo flujo garantiza el desempeño organizacional. El planteamiento esencial es que existen unos factores estratégicos -liderazgo y estilo directivo, clima organizacional, tamaño y habilidades del perso-

Ilustración 3. El modelo de la congruencia organizacional ${ }^{13}$

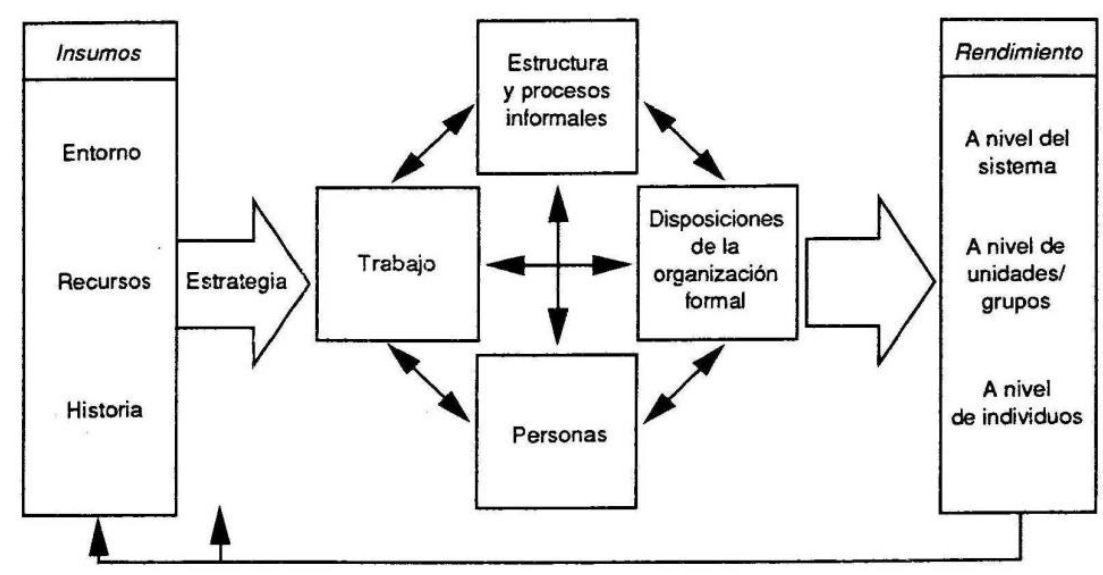

13 Fuente: Nadler \& Tushmann (1997) 
nal, ambiente, tecnología y estrategia-, y unos factores estructurales -configuraciones, complejidad, formalización, centralización, incentivos y coordinación y control-, cuya alineación garantizarán una mayor efectividad (eficacia + eficiencia) y la perdurabilidad de la organización (Ilustración 4).

La teoría de la multi-contingencia estructural es operacionalizada a través de una metodología de matrices en un sistema de coordenadas con cuatro cuadrantes, en donde cada uno de ellos comprende una tipología estructural. Empíricamente, el predominio del análisis organizacional en un cuadrante indicará organizaciones ajustadas (Burton, Obel, \& DeSanctis, 2011).

Ilustración 4. Modelo de la multi-contingencia organizacional $^{14}$

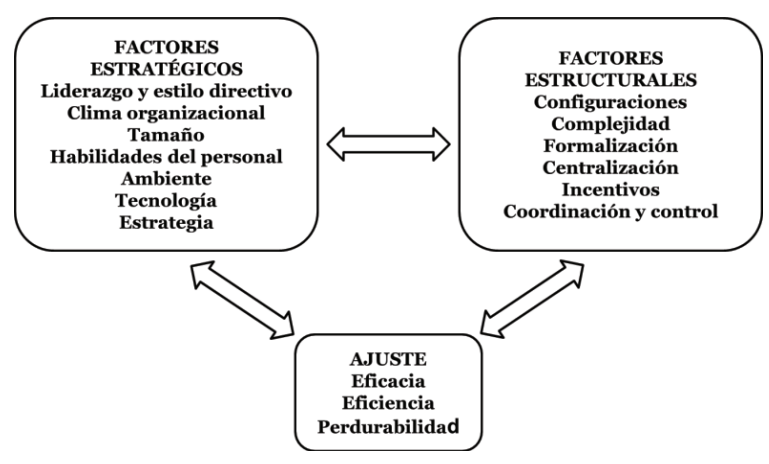

Finalmente, una característica interesante de la teoría de la co-alineación estratégica es la propiedad de equifinalidad que le es inherente (Doty et al., 1993; Ketchen, Thomas, \& Snow, 1993; Meyer, Tsui, \& Hinings, 1993). La equifinalidad es un principio derivado de la teoría de sistemas que establece que "los sistemas pueden alcanzar el mismo estado final desde diferentes condiciones y por diferentes rutas de desarrollo" (Katz \& Kahn, 1966, 28). Traducido al campo organizacional, sugiere la posibilidad de llegar a objetivos comunes a partir de diferentes estrategias (Kast \& Rosenzweig, 1972). Por lo tanto, en el campo de la congruencia estructural, la equifinalidad es usada para significar: (i) la existencia de múltiples condiciones de configuración tales como diversidad ambiental, diferentes patrones de recursos, varios procesos de transformación interna y variados arreglos de los componentes estructurales endógenos, y (ii) la existencia de más de un modo de lograr el desempeño a través de las diferentes configuraciones (Miller, 1986, 1987; Veliyath \& Srinivasan, 1995).

\section{Conclusiones}

Con la introducción del funcionalismo organizacional a comienzos del siglo XX, especialmente con los aportes del ingeniero francés Henri Fayol y con los desarrollos posteriores realizados por la escuela neoclásica, se introdujo en la administración los conceptos y elementos a considerar en la conformación y funcionamiento de la organización (Pfeffer, 1982).

No obstante su trascendencia e impacto en la forma de dividir y coordinar el trabajo de los agentes organizacionales, su consideración de la organización se limitó al análisis de las variables internas que afectan la eficiencia, en una visión de órgano cerrado que no considera las afectaciones ambientales.

El surgimiento de la teoría de sistemas y su aplicación a la administración, produjo un replanteamiento en esa visión al reconocer que el ambiente tiene un rol de incidencia manifiesto en la forma de configurar las organizaciones (Kast \& Rosenzweig, 1972; Katz \& Kahn, 1966). Los postulados de esta teoría motivaron una corriente de estudios de naturaleza determinista que centra su análisis en las propiedades estructurales que están influenciadas por las condiciones del ambiente y que inciden en el comportamiento de las organizaciones (Astley \& Van de Ven, 1983). En este marco emergen consecuentemente dos de las principales teorías situacionales: La teoría de la contingencia estructural y la teoría de la coalineación estratégica.

Siguiendo a Astley \& Van de Ven (1983), en su clasificación de las escuelas de pensamiento organizacional en el nivel micro (organización individual), la orientación determinista introdujo a las teorías del sistema estructural, contingencia y co-alineación,

14 Fuente: Burton \& Obel (2004). 
como los dos principales referentes en el campo del análisis y diseño organizacional.

La teoría de la contingencia estructural enfatizó la importancia que tiene el ambiente sobre la estructura organizacional, indicando que las empresas se configuran de diversas maneras según las influencias externas recibidas (Donaldson, 2001). Así, en el proceso de diferenciación e integración organizacional ya no bastará únicamente la articulación de las variables internas, sino además su correspondencia con el entorno en el que opera la empresa (Lawrence \& Lorsch, 1967), circunstancia que dará lugar a diferentes formas organizacionales.

No obstante los importantes aportes de la teoría de la contingencia estructural, su explicación del diseño organizacional para lograr un mejor desempeño quedo eclipsada y reducida (Donaldson, 1987). Frente a esto, algunos académicos se interesaron en determinar cómo, ante las influencias ambientales, las variables estructurales endógenas se acoplan para proveer un mayor desempeño organizacional, surgiendo la teoría de la co-alineación estratégica o del fit que ha entregado los referentes para determinar las condiciones del ajuste estructural (Drazin \& Van de Ven, 1985; Ensign, 2001; Venkatraman \& Camillus, 1984).

Estas dos teorías tienen importantes implicaciones teóricas y prácticas. En cuanto a las implicaciones teóricas, han sido el principal referente en la teoría de la organización para el desarrollo de diferentes enfoques acerca del análisis y diseño organizacional (Burton \& Obel, 2004; Galbraith, 1973; Mintzberg, 1979). En cuanto a las implicaciones prácticas, ofrecen un marco para la comprensión del fenómeno de estructuración organizacional, en especial en Colombia en donde aún existe algún grado de informalidad en el diseño organizacional (Marin-Idárraga, 2012b).
A modo de síntesis, en la Tabla 4 y en la Tabla 5, se presenta un resumen de los principales autores analizados en el presente artículo.

Tabla 4. Principales representantes e ideas de la teoría de la contingencia estructural ${ }^{15}$

\begin{tabular}{|c|c|}
\hline Autor & Aporte \\
\hline Burns \& Stalker (1961) & $\begin{array}{l}\text { Las decisiones de diseño organi- } \\
\text { zacional llevan a organizaciones } \\
\text { estructuradas de manera mecánica } \\
\text { (Alta especialización, formalización } \\
\text { y centralización) u orgánica (Baja } \\
\text { especialización, formalización y cen- } \\
\text { tralización). }\end{array}$ \\
\hline $\begin{array}{c}\text { Woodward (1965), Thompson } \\
\text { (1967) y Perrow (1967) }\end{array}$ & $\begin{array}{l}\text { La tecnología afecta de manera con- } \\
\text { tingente la forma en que la organiza- } \\
\text { ción divide y coordina el trabajo. }\end{array}$ \\
\hline Lawrence \& Lorsh (1967) & $\begin{array}{l}\text { El diseño de la organización depende } \\
\text { de fuerzas ambientales que implican } \\
\text { decisiones de diferenciación e inte- } \\
\text { gración del trabajo. }\end{array}$ \\
\hline $\begin{array}{l}\text { Blau (1970), Meyer (1971; } \\
\text { 1972) y Child (1972b; 1973) }\end{array}$ & $\begin{array}{l}\text { El tamaño es una variable contingente } \\
\text { del diseño organizacional. A mayor } \\
\text { tamaño, mas presión hacia la diferen- } \\
\text { ciación, la especialización, la formali- } \\
\text { zación y la descentralización. }\end{array}$ \\
\hline $\begin{array}{l}\text { Chandler (1962), Channon } \\
\text { (1973), Rumelt (1974) y Dyas } \\
\text { \& Thangheiser (1976) }\end{array}$ & La estructura sigue a la estrategia. \\
\hline $\begin{array}{l}\text { Hinnings et al. (1967), Pugh } \\
\text { \& Pheysey (1972), Pugh et al. } \\
\text { (1963), Pugh et al. (1968) y } \\
\text { Pugh et al. (1969a; 1969b) }\end{array}$ & $\begin{array}{l}\text { Grupo Aston inglés que, con base } \\
\text { en la burocracia weberiana, estudió } \\
\text { y caracterizó las diferentes variables } \\
\text { internas que inciden en el diseño } \\
\text { de la organización (especialización, } \\
\text { diferenciación, formalización, centra- } \\
\text { lización). }\end{array}$ \\
\hline Hage (1965) & $\begin{array}{l}\text { El diseño organizacional comprende } \\
\text { una axiomática con base en los sig- } \\
\text { nificados y fines de la organización. }\end{array}$ \\
\hline
\end{tabular}

15 Fuente: Elaboración propia.

16 Fuente: Elaboración propia. 
Tabla 5. Principales representantes e ideas de la teoría de la co-alineación estratégica ${ }^{16}$

\begin{tabular}{|c|c|}
\hline Autor & Aporte \\
\hline $\begin{array}{l}\text { Venkatraman \& } \\
\text { Camillus (1984) } \\
\text { y Venkatraman } \\
\quad(1989)\end{array}$ & $\begin{array}{l}\text { Existen diferentes factores contingentes externos } \\
\text { que inciden en la forma como la organización di- } \\
\text { vide y coordina el trabajo. La capacidad de alinear } \\
\text { las variables internas de la estructura con dichos } \\
\text { factores, garantizará un mayor desempeño. }\end{array}$ \\
\hline $\begin{array}{l}\text { Drazin \& Van de } \\
\text { Ven (1985) }\end{array}$ & $\begin{array}{l}\text { El Ajuste entre las variables estructurales y las } \\
\text { fuerzas ambientales se da en las perspectivas de } \\
\text { selección, interacción y sistemas. }\end{array}$ \\
\hline Donaldson (1987) & $\begin{array}{l}\text { Modelo SARFIT: Los factores contingentes produ- } \\
\text { cen un desajuste en la organización Ilevándola a un } \\
\text { bajo rendimiento, lo cual ocasiona necesariamente } \\
\text { un cambio estructural para alcanzar el ajuste y me- } \\
\text { jorar el desempeño. }\end{array}$ \\
\hline $\begin{array}{l}\text { Venkatraman } \\
\text { (1990) }\end{array}$ & $\begin{array}{l}\text { En la investigación empírica, el ajuste se explica y } \\
\text { comprende desde tres perspectivas: interaccionis- } \\
\text { ta, desviación de un perfil y co-variación. }\end{array}$ \\
\hline $\begin{array}{c}\text { Nadler \& Tush- } \\
\text { mann (1997) }\end{array}$ & $\begin{array}{l}\text { Modelo de la congruencia organizacional. Desde } \\
\text { un enfoque de sistemas, explica que los factores } \\
\text { contingentes ambientales inducen un re-acoda- } \\
\text { miento de la estructura formal e informal para bus- } \\
\text { car un mayor desempeño. }\end{array}$ \\
\hline $\begin{array}{l}\text { Ensign (2001) y } \\
\text { Sillince (2005) }\end{array}$ & $\begin{array}{l}\text { Los factores contingentes (ambiente y estrategia) } \\
\text { influyen en la organización presionando un dimen- } \\
\text { sionamiento de su estructura para alcanzar un ma- } \\
\text { yor desempeño. }\end{array}$ \\
\hline $\begin{array}{l}\text { Burton \& Obel } \\
\quad(2004)\end{array}$ & $\begin{array}{l}\text { Modelo de la multi-contingencia organizacional. } \\
\text { La organización posee unos factores estratégicos } \\
\text { y unos factores estructurales, cuyo acople, garanti- } \\
\text { zará un mejoramiento en la eficiencia y la eficacia. }\end{array}$ \\
\hline
\end{tabular}

\section{Referencias}

Anderson, T., \& Warkov, S. (1961). "Organizational size and functional complexity: a study of differentiation in hospitals". En: American Sociological Review, 26: 23-28.

Astley, W. G., \& Van de Ven, A. H. (1983). "Central perspectives and debates in organization theory". En: Administrative Science Quarterly, 28(2): 245-273.

Blau, P. (1970). "A formal theory of differentiation in organizations". En: American Sociological Review, 35(2): 201-218.

Burns, T. \& Stalker, G. (1961). The Management of Innovation. London: Tavistock.
Burton, R. \& Obel, B. (2004). Strategic organizational diagnosis and design: The dynamics of fit. Massachusetts: Kluwer Academic Publishers.

Burton, R., Obel, B. \& DeSanctis, G. (2011). Organizational design: a step-by-step aprroach. New York: Cambridge University Press.

Chandler, A. (1962). Strategy and structure: chapters in the history of the American industrial enterprise. Washington: MIT Press.

Channon, D. F. (1973). The strategy and structure of British enterprise. London: Macmillan Press.

Child, J. (1972a). "Organizational structure, environment and performance: the role of strategic choice". En: Sociology, 6(1): 1-22.

Child, J. (1972b). "Organization structure and strategies of control: a replication of the Aston study". En: Administrative Science Quarterly, 17: 163-177.

Child, J. (1973). "Strategies of control and organizational behavior". En: Administrative Science Quarterly, 18: 1-17.

Christensen, M. \& Raynor, M. (2003). "Why hard-nosed executives should care about mangement theory". En: Harvard Business Review, September: 4-10.

Courtright, J., Fairhurst, G., \& Rogers, E. (1989). "Interaction patterns in organic and mechanistic systems". En: Academy of Management Journal, 32(4): 773-802.

Dalton, D. R., Todor, W. D., Spendolini, M. J., Fielding, G. J., \& Porter, L. W. (1980). "Organization structure and performance: A critical review". En: Academy of Management Review, 5(1): 49-64.

Dewar, R. \& Werbel, J. (1979). "Universalistic and contingency predictions of employee satisfaction and conflict". En: Administrative Science Quarterly, 24: 426-448.

Donaldson, L. (1987). "Strategy and structural adjustment to regain fit and performance in defence of contingency theory". En: Journal of Management Studies, 24(1): 1-24.

Donaldson, L. (2001). The contingency theory of organizations. California: Sage Publications.

Doty, D., Glick, W. \& Huber, G. (1993). "Fit, equifinality, and organizational effectiveness: a test of two configurational theories". En: Academy of Management Journal, 36(6): 1196-1250.

Drazin, R., \& Van de Ven, A. H. (1985). "Alternative forms of fit in contingency theory". En: Administrative Science Quarterly, 30(4): 514-539.

Dyas, G. P. \& Thanheiser, H. (1976). The emerging European enterprise: Strategy and structure in French and German industry. London: Macmillan Press.

Ensign, P. (2001). "The concept of fit in organizational research". En: International Journal of Organization, Theory \& Behavior, 4(3): 287-306.

Fayol, H. (1949). General and Industrial Management. London: Pitman.

Fegh-hi, N. (2010). "Strategic structure for organizational performance". En: International Journal of Management \& Innovation, 2(2): 9-23.

Fry, L. W. (1982). "Technology-structure research: Three critical issues". En: Academy of Management Journal, 25(3): 532-552.

Fry, L. W., \& Smith, D. A. (1987). "Congruence, contingency, and theory building". En: Academy of Management Review, 12(1): 117-132.

Galbraith, J. R. (1973). Designing Complex Organizations. Reading, MA: Addison-Wesley.

Grinyer, P. J. \& Yasai-Ardekani, M. (1980). "Dimensions of organizational structure: a critical replication". En: Academy of Management Journal, 23: 405-421. 
Hage, J. (1965). "An axiomate theory of organizations". En: Administrative Science Quarterly, 10: 289-320.

Hall, R. (1962). "Intraorganizational structural variation: application of the bureaucratic model". En: Administrative Science Quarterly, 7: 295-308.

Hinings, C.R., Pugh, D.S., Hickson, D.J., \& Turner, C. (1967). "An approach to the study of bureaucracy". En: Sociology, 1:61-72.

Kast, F. E., \& Rosenzweig, J. E. (1972). "General system theory: Applications for organization and management”. En: Academy of Management Journal, 15(4): 447-465.

Katz, D \& Kahn, R. (1966).The Social Psychology of Organizations. New York: John Wiley \& Sons, Inc.

Ketchen, D. J., Thomas, J. B., \& Snow, C. C. (1993). "Organizational configurations and performance: a comparison of theoretical approaches". En: Academy of Management Journal, 36(6): 1278-1313.

Lawrence, P. \& Lorsch, J. (1967). "differentiation and integration in complex organizations". En: Administrative Science Quarterly, 12(1): $1-47$.

Liao, C., Chuang, S., \& To, P. (2011). "How knowledge management mediates the relationship between environment and organizational structure". En: Journal of Business Research, 64(7): 728-736.

Marín-Idárraga, D. A. (2012a). "Consideraciones epistemológicas en torno al carácter científico de la administración". En: Innovar, 22(46): 39-52.

Marín-Idárraga, D. A. (2012b). "Estructura organizacional y sus parámetros de diseño: análisis descriptivo en pymes industriales de Bogotá". En: Estudios Gerenciales, 28(123): 43-64.

McCaskey, M. (1974). "An introduction to organizational design". En: California Management Review, 17(2): 13-20.

Menguc, B., \& Auh, S. (2010). "Development and return on execution of product innovation capabilities: The role of organizational structure". En: Industrial Marketing Management, 39(5): 820-831.

Meyer, A. D., Tsui, A. S., \& Hinings, C. R. (1993). "Configurational approaches to organizational analysis". En: Academy of Management Journal, 36(6): 1175-1195.

Meyer, M. (1971). "Some constraints in analyzing data on organizations structures". En: American Sociological Review, 36: 294-297.

Meyer, M. (1972). "Size and structure of organizations: a causal analysis". En: American Sociological Review, 37: 434-440.

Miller, D. (1987). "The genesis of configuration". En: The Academy of Management Review, 12(4): 686-701.

Miller, D. (1986). "Configurations of strategy and structure: towards a synthesis". En: Strategic Management Journal, 7(3): 233-249.

Mintzberg, H. (1979). The structuring of organizations: A synthesis of the research. Englewood Cliffs, NY: Prentice-Hall.

Nadler, D. A. \& Tushman, M. L. (1997). Competing by design: the power of organizational architecture. New York: Oxford University Press.

Nadler, D. A., \& Tushman, M. L. (1999). "The organization of the future: Strategic imperatives and core competencies for the 21st century". En: Organizational Dynamics, 28(1): 45-60.

Perrow, Ch. (1967). "A framework for the comparative analysis of organizations". En: American Sociological Review, 32(2): 194-208.

Pfeffer, J. (1982). Organizations and organization theory. Cambridge: Ballinger Publishing.
Pugh, D. S. \& Pheysey, D.C. (1972). "Some developments in the study of organizations". En: Administrative Science Quarterly. 17: 273-276.

Pugh, D. S., Hickson, D. J., Hinnings, C. R. \& Turner, C. (1968). "Dimensions of organizational structure". En: Administrative Science Quarterly, 13: 65-105.

Pugh, D. S., Hickson, D. J. \& Hinnings, C. R. (1969a). "The context of organization structures". En: Administrative Science Quarterly, 14: 91-114.

Pugh, D. S., Hickson, D. J. \& Hinnings, C. R. (1969b), "An empirical taxonomy of work organizations". En: Administrative Science Quarterly, 14: 115-126.

Pugh, D.S. Hickson, D.J., Hinings, C.R., Macdonald, K.M., Turner, C., \& Lupton, T. (1963). "A conceptual scheme for organizational analysis”. En: Administrative Science Quarterly, 8: 289-315.

Rapert, M., \& Wren, B. (1998). "Reconsidering organizational structure: A dual perspective of frameworks and process". En: Journal of Managerial Issues, 10(3): 287-302.

Reimann, B. C. (1973). "On the dimensions of bureaucratic structure: an empirical reappraisal". En: Administrative Science Quarterly, 18: 462-476.

Rumelt, R.P. (1974), Strategy, Structure, and Economic Performance. Boston, MA: Division of Research, Harvard Business School.

Sahay, Y. P., \& Gupta, M. (2011). "Role of organization structure in innovation in the bulk-drug industry". En: Indian Journal of Industrial Relations, 46(3): 450-464.

Santra, T. \& Giri, V. N. (2008). "Effect of organizational structure on organizational effectiveness through face-to-face communication". En: ICFAI Journal of Organizational Behavior, 7(2): 28-38.

Schoonhoven, C. B. (1981). "Problems with contingency theory: Testing assumptions hidden within the language of contingency theory". En: Administrative Science Ouarterly, 26: 349-377.

Sexton, W. (2005). Teorías de la Organización (9 reimp). México: Trillas.

Sillince, J. A. (2005). "A contingency theory of rhetorical congruence". En: Academy of Management Review, 30(3): 608-621.

Sutton, R. I. \& Staw, B. M. (1995). "What theory is not". En: Administrative Science Quarterly, 40(3): 371-384.

Taylor, F. W. (1911). The principles of scientific management. New York: Harper \& Brothers.

Thompson, J. (1967). Organizations in action. New York: McGraw Hill.

Tosi, H. \& Platt, H. (1967). "Administrative ratios and organizational size". En: Academy of Management Journal, 10: 161-168.

Veliyath, R. \& Srinivasan, T. C. (1995). "Gestalt approaches to assessing strategic coalignment: a conceptual integration". En: British Journal of Management, 6(3): 205-219.

Venkatraman, N. (1989). "The concept of fit in strategy research: Toward verbal and statistical correspondence". En: Academy of Management Review, 14(3): 423-444.

Venkatraman, N. (1990). "Performance implications of strategic coalignment: A methodological perspective". En: Journal of Management Studies, 27(1): 19-41.

Venkatraman, N. \& Camillus, J. C. (1984). "Exploring the concept of "fit" in strategic management". En: Academy of Management Review, 9(3): 513-525.

Weber, M. (1947). The theory of social and economic organization. NewYork: Free Press.

Weick, K. E. (1989). "Theory construction as disciplined imagination". En: Academy of Management Review, 14: 516-531. 
Williamson, O. (1970). Corporate control and business behavior. Englewoods Cliffs: Prentice Hall.

Woodward, J. (1965). Industrial organization: theory and practice. London: Oxford University Press.

Zapata, G. \& Mirabal, A. (2013). "Contingencia, voluntarismo y Strategic Choice: un análisis teórico de sus enfoques sobre el entorno y el diseño de la organización”. En: Universidad y Empresa, 24: 119-139.

Zapata, G., Mirabal, A. \& Hernández, A. (2009). "Modelo teórico conceptual de la estructura organizativa: un análisis contingente". En: Ciencia y Sociedad, 34(4): 618-640. 